\title{
Expression of STAT3 in non-small cell lung cancer and its clinical significance
}

\author{
Hongcheng Wang ${ }^{1^{*}}$, Xiu Lan ${ }^{2}$, Benyan Song $^{3}$, Caixia Xin $^{1}$, Chao Wang $^{1}$, Hong Dai $^{1}$ \\ ${ }^{1}$ Department of Respiratory Medicine, The Affiliated Hospital of Luzhou Medical College, Luzhou, China; \\ *Corresponding Author: hongchengw49@yahoo.com.cn \\ ${ }^{2}$ Department of Respiratory Medicine, The Center Hospital of Lishui City, Lishui, China \\ ${ }^{3}$ Department of Respiratory Medicine, The Affiliated Hospital of Pan Zhi-Hua Medical College, Panzhihua, China
}

Received 12 January 2013; revised 25 February 2013; accepted 6 March 2013

Copyright (C) 2013 Hongcheng Wang et al. This is an open access article distributed under the Creative Commons Attribution License, which permits unrestricted use, distribution, and reproduction in any medium, provided the original work is properly cited.

\section{ABSTRACT}

Signal transducer and activator of transcription 3(STAT3) plays an important role in tumor occurrence and development. Here we attempt to evaluate the clinical diagnostic value of STAT3 as a potential tumor marker by detecting the expression of STAT3 in blood of non-small cell lung cancer (NSCLC) patients. Immunohistochemistry was applied to detect STAT3 protein in A549 cells and MRC- 5 cell, real time PCR was applied to detect STAT3 mRNA and ELISA was applied to detect the expression of STAT3 protein in NSCLC patients, benign lung disease group and healthy controls. The expression levels of STAT3 mRNA and STAT3 protein in A549 cells and NSCLC patients were significantly higher $(P<0.01)$. The areas under ROC curves (Az) of STAT3 mRNA and STAT3 protein were 0.870 and 0.860 respectively, which had no significant difference $(P>0.05)$. When comparing the $A z$ of STAT3 protein with CEA, CA125 and CYFRA21-1 respectively, there was no significant difference $(P>0.05)$. And we compared the $A z$ of the four joint detection (STAT3 + CEA + CA125 + CYFRA21-1) with the three joint detection (CEA + CA125 + CYFRA21-1), 0.930 and 0.841 , respectively, $P<0.05$. It appears that the diagnostic value and accuracy of STAT3 is equivalent with CEA, CA125, and CYFRA21-1 in NSCLC, and to joint with other three can achieve higher diagnosis value.

Keywords: Signal Transducer and Activator of Transcription 3; Non-Small Cell Lung Cancer; Tumor Marker; ROC Curve; Diagnostic Value

\section{INTRODUCTION}

Currently, lung cancer has become the leading cause of death. In recent years, people had extensively studied on its development, invasion and metastasis mechanism. STAT3 is a kind of key nuclear transcription factor, and there is a lot of research about it lately. It is widely expressed in different types of cells and tissues to participate in the functions of regulation on cell's growth, malignant transformation and apoptosis. Study found that in head and neck cancer, NSCLC and other malignant tumors and cell lines, STAT3 has abnormal expression and activity increased. It suggests that abnormal expression of STAT3 plays an important role in tumor occurrence and development. In this study, Real time PCR, immunohistochemistry, and ELISA were applied to analyze the abundance of STAT3 mRNA and the expressions of STAT3 protein in human lung adenocarcinoma A549 cell line and the blood of NSCLC patients for further exploring of STAT3's diagnostic value in NSCLC, and they also provided a theoretical and experimental basis for the clinical applications of STAT3.

\section{MATERIALS AND METHODS}

\subsection{Main Reagents}

TRIZOL reagent was purchased from Beijing TIANGEN company; RT Buffer, dNTP Mixture, Super-RI, RTEnhancer, Oligo (dT) 18 and ReverTra Ace were purchased from Chengdu Bo Ruike biotech companies; Taq enzyme, dNTP were purchased from Takara Bio Inc.; PCR primers were purchased from Shanghai Bio-Engineering Co., Ltd.; enzyme-linked immunosorbent assay kit of STAT3, CEA, CA125, and CYFRA21-1 in human were purchased from RD US companies; Rabbit AntiHuman STAT3 Polyclonal Antibody was purchased from Bioworld technology companies. 


\subsection{Sources}

Human lung adenocarcinoma A549 cells, normal human embryonic lung MRC-5 cell line were provided by the Central Laboratory of Luzhou Medical College. Clinical information was gathered from Luzhou Medical School Affiliated Hospital, lung cancer group have 40 patients, the median age was 54 years old, both NSCLC (22 cases of squamous cell carcinoma, 18 cases of adenocarcinoma; 13 cases of well-differentiated, 17 cases of moderately differentiated, 10 cases of poorly differentiated; 23 cases of lymph node metastasis, 17 cases without lymph node metastasis), and have not received chemotherapy, radiotherapy, biological therapy and other special treatment, and also no other previous malignancies in history. There were 40 cases of benign lung disease group, the median age was 55 years old, who were clinical diagnosis confirmed and rule out lung cancer. And also selected 40 healthy volunteers as healthy controls, the median age was 51 years old.

\subsection{Detection of STAT3's Expression}

\subsubsection{Immunohistochemistry}

The human lung adenocarcinoma A549 cells and normal human embryonic lung MRC-5 cells were inoculated on cover slips for cells seeded respectively, then washed the specimens with PBS after 5 days, next used 4\% paraformaldehyde to fix for $20 \mathrm{~min}$, incubated with $0.5 \%$ Triton X-100 for $20 \mathrm{~min}$, and then done the remaining operating according to kit instructions.

\subsubsection{Real Time PCR}

1) Extracted total RNA from cells and peripheral blood (according to the principles of random, selected 10 patients as samples from lung cancer group, benign lung disease group and healthy controls, and distilled $4 \mathrm{ml}$ fasting blood from their peripheral venous), measured OD260/OD280 (R) value with trace nucleic acid quantitative instrument, and then reversed transcript the one whose $\mathrm{R}$ value was between 1.8 to 2.0 . 2) Primer design: used Primer 5.0 software to design, and used GAPDH as internal control, primer sequences and amplified fragment (Table 1). 3) Real time PCR detection: established fluorescence quantitative PCR reaction system with SYBR Green I: upstream and downstream primers, $1 \mu \mathrm{l}, 2 \times$ Mix $12.5 \mu \mathrm{l}$, respectively (including Taq polymerase, dNTP, $\mathrm{Mg}^{2+}$, SYBRGreen I, etc.); doubledistilled water, $8.5 \mu \mathrm{l}$, cDNA $2 \mu \mathrm{l}$. Conditions for PCR reaction: $95^{\circ} \mathrm{C} 2 \mathrm{~min} ; 95^{\circ} \mathrm{C} 5 \mathrm{~s}, 61.4^{\circ} \mathrm{C} 20 \mathrm{~s}$ (STAT3, GAPDH all were $\left.58.7^{\circ} \mathrm{C}\right), 72^{\circ} \mathrm{C} 15 \mathrm{~s}, 40$ cycles in total, collect fluorescence at the second step of the cycle, and analyzed the melting curve at the end of amplification. 4) Quantification for the mRNA of target gene: established standard curve, and took the template's amount of GAPDH
Table 1. Primers and sequencing.

\begin{tabular}{ccc}
\hline Primer & Array & Length (bp) \\
\hline GAPDH & 5'-ATgCTggCgCTgAgTACgTC-3' & 133 \\
& 5'-ggTCATgAgTCCTTCCACgATA-3' & \\
STAT3 & 5'-CCTgAAgCTgACCCAggTAg-3' & 237 \\
& 5-TTCCAAACTgCATCAATgAATC-3' \\
\hline
\end{tabular}

mRNA as reference to calculate the relative expression of STAT3 mRNA. Apply the relative quantitative method Pfaffl to compare the differences of various gene expressions.

\subsubsection{Enzyme-Linked Immunosorbent Assay (ELISA)}

All personnel enrolled are distilled $4 \mathrm{~mL}$ fasting blood with procoagulant tube from peripheral venous, centrifugated 1500 turn for 15 minutes, then collected the upper serum and injected them into $2 \mathrm{ml}$ EP tube, frozen at $-80^{\circ} \mathrm{C}$ until to be used. Operate according to kit instructions.

\subsubsection{Judgment of Immunohistochemical Results}

Brown or brown granules appearing in the cells was positive, randomly selected five positive staining area from each positive slices, photograph under 200 magnification, evaluated the MOD values of each photo with the image analysis software IPP6.0, and then took the mean MOD of the five horizons in each slice for analysis and comparison.

\subsubsection{Statistical Treatment}

SPSS17.0 was used for statistical analysis. Variable data were expressed as mean $\pm \mathrm{SD}(\overline{\mathrm{x}} \pm \mathrm{s})$ and analyzed using single-factor analysis of variance. The diagnostic value of STAT3 was evaluated with ROC curve, and the diagnostic accuracy was compared by $\mathrm{Z}$ test. The value of $P<0.05$ was considered to be statistically significant.

\section{RESULTS}

\subsection{STAT3's Expression}

\subsubsection{STAT3's Expression in Cells}

The expression of STAT3 mRNA in A549 cells was significantly higher than in MRC-5 cells $(P<0.01)$ (Table 2). In A549 cells, a few positive signal of STAT3 protein line in the nucleus, but mainly line in the cytoplasm, which showed a brown-yellow or brown granular staining, and its expression is weak in MRC-5, showing yellow color (Figures 1 and 2). The expression of STAT3 protein in A549 cell was significantly higher than in MRC-5 cells (Table 3). 
Table 2. Expression of STAT3 mRNA in A549 and MRC-5 cells $(\overline{\mathrm{x}} \pm \mathrm{s})$.

\begin{tabular}{ccccc}
\hline Groups & Number of cases & STAT3 protein & $F$ value & $P$ value \\
\hline A549 Cells & 15 & $6.96 \pm 3.37$ & & \\
MRC-5 Cells & 5 & $1.01 \pm 0.19$ & 9.008 & 0.001 \\
\hline
\end{tabular}

Table 3. Expression of STAT3 protein in A549 and MRC-5 cells $(\overline{\mathrm{x}} \pm \mathrm{s})$.

\begin{tabular}{ccccc}
\hline Groups & Number of cases & STAT3 protein & $F$ value & $P$ value \\
\hline A549 Cells & 20 & $0.133 \pm 0.067$ & & \\
MRC-5 Cells & 20 & $0.049 \pm 0.010$ & 2.995 & 0.001 \\
\hline
\end{tabular}

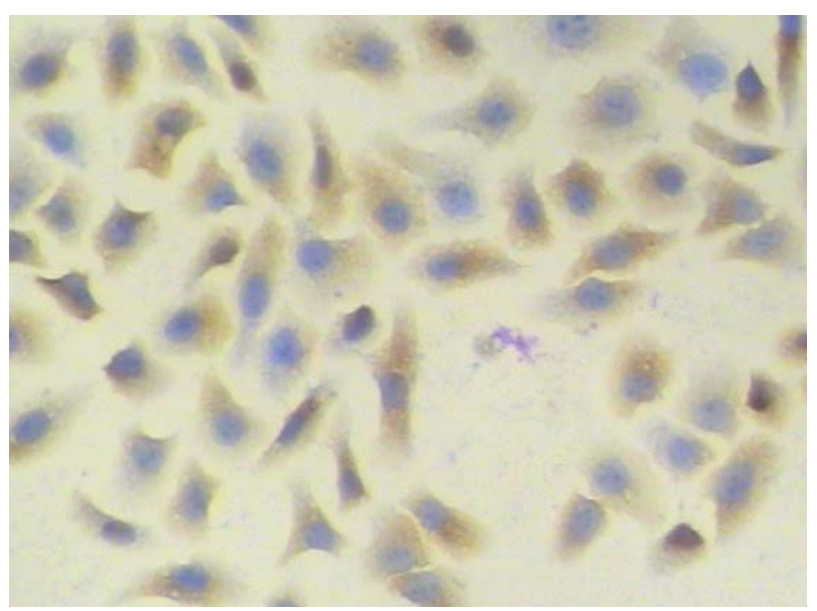

Figure 1. STAT3 protein in human lung adenocarcinoma A549 cells $(\mathrm{PV}$ method $\times 200)$.

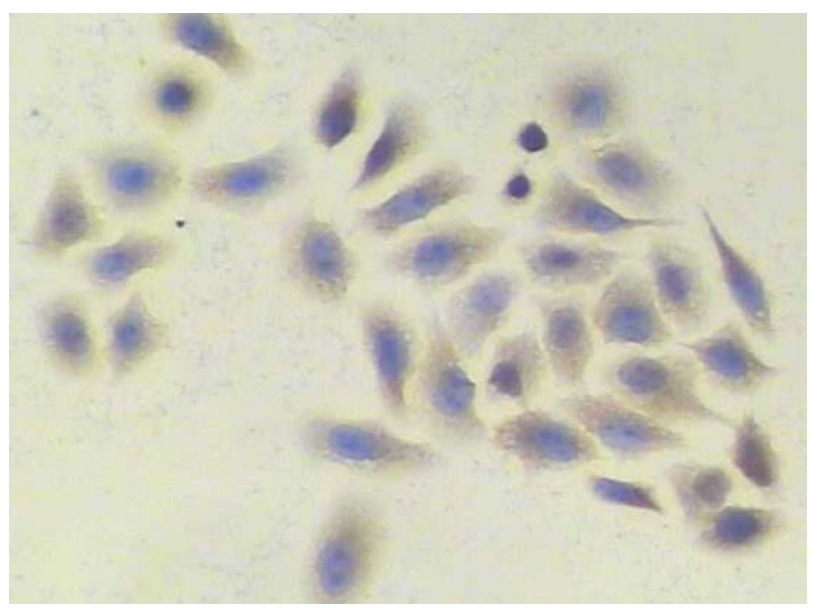

Figure 2. STAT3 protein in human embryonic lung MRC-5 cells (PV method $\times 200)$.

\subsubsection{STAT3's Expression in the Blood}

The expression of STAT3 mRNA and STAT3 protein in the lung cancer group were significantly higher than in the normal benign lung disease group and healthy controls $(P<0.01)$ and their expression were no significant difference in benign lung disease group and healthy con- trols $(P>0.05)$ (Tables 4 and 5). In which, STAT3 protein's expression was unrelated with pathological type or whether there is lymph node metastasis $(P>0.05)$, but it's related with the degree of histological differentiation $(P<0.01)$. The lower the degree of differentiation, the higher the level of STAT3's expression (Table 6).

\subsection{The Diagnostic Value of STAT3 in NSCLC}

The Az of STAT3 mRNA and STAT3 protein were 0.870 and 0.860 . Comparison between the two Az, $\mathrm{Z}=$ $0.002, P=0.500$, no statistically significant difference $(P$

Table 4. Comparison of STAT3 mRNA's expression in peripheral blood samples of each group $(\bar{x} \pm s)$.

\begin{tabular}{ccccc}
\hline Groups & $\begin{array}{c}\text { Number of } \\
\text { cases }\end{array}$ & $\begin{array}{c}\text { STAT3 } \\
\text { protein }\end{array}$ & $F$ value & $P$ value \\
\hline Lung cancer & 10 & $2.92 \pm 1.26^{\mathrm{a}}$ & & \\
Benign lung disease & 10 & $1.39 \pm 1.10^{\mathrm{b}}$ & 9.008 & 0.001 \\
Healthy controls & 10 & $1.00 \pm 0.79$ & & \\
\hline
\end{tabular}

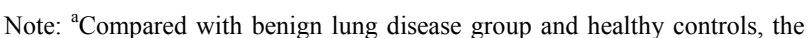
difference were statistically significant, $P<0.05$; ${ }^{\mathrm{b}}$ Compared with the healthy controls, no statistically significant difference, $P>0.05$.

Table 5. Comparison of STAT3 protein's expression in serum samples of each group $(\overline{\mathrm{x}} \pm \mathrm{s})$.

\begin{tabular}{ccccc}
\hline Groups & $\begin{array}{c}\text { Number } \\
\text { of cases }\end{array}$ & $\begin{array}{c}\text { STAT3 protein } \\
(\rho \mathrm{g} / \mathrm{ml})\end{array}$ & $F$ value & $P$ value \\
\hline Lung cancer & 40 & $118.28 \pm 34.65^{\mathrm{a}}$ & & \\
Benign lung disease & 40 & $72.51 \pm 17.73^{\mathrm{b}}$ & 50.541 & 0.00 \\
Healthy controls & 40 & $69.80 \pm 15.70$ & & \\
\hline
\end{tabular}

Note: ${ }^{a}$ Compared with benign lung disease group and healthy controls, the difference were statistically significant, $P<0.01$; ${ }^{\mathrm{b}}$ Compared with the healthy controls, no statistically significant difference, $P>0.05$.

Table 6. The relationships of Serum STAT3 protein's expression and clinical pathological factors.

\begin{tabular}{|c|c|c|c|c|}
\hline Groups & $\begin{array}{l}\text { Number } \\
\text { of cases }\end{array}$ & $\begin{array}{l}\text { STAT3 protein } \\
(\rho \mathrm{g} / \mathrm{ml})\end{array}$ & $F$ value 1 & $P$ value \\
\hline \multicolumn{5}{|l|}{ Pathological type } \\
\hline Squamous cell carcinoma & 22 & $107.75 \pm 30.08$ & 0.322 & 0.574 \\
\hline Adenocarcinoma & 18 & $114.64 \pm 46.29$ & & \\
\hline \multicolumn{5}{|l|}{ Degree of differentiation } \\
\hline Poorly differentiated & 10 & $149.74 \pm 37.90^{\mathrm{a}}$ & & \\
\hline Differentiated & 17 & $100.39 \pm 33.13^{\mathrm{b}}$ & 10.644 & 0.00 \\
\hline Well-differentiated & 13 & $94.61 \pm 20.39$ & & \\
\hline \multicolumn{5}{|l|}{ Lymph node metastasis } \\
\hline With lymph node metastasis & 23 & $120.31 \pm 45.16$ & 3.607 & 0.065 \\
\hline $\begin{array}{l}\text { Without lymph node } \\
\text { metastasis }\end{array}$ & 17 & $98.05 \pm 19.66$ & & \\
\hline
\end{tabular}

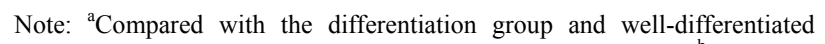
group, the difference were statistically significant, $P<0.05$; ${ }^{\mathrm{b}}$ Compared with well-differentiated group, no statistically significant difference, $P>$ 0.05 . 
$>0.05$ ). Compared the Az of STAT3 protein with CEA, CA125 and CYFRA21-1 respectively, no statistically significant difference $(P>0.05)$ (Figure 3, Table 7). Select the best cut-off point according to Youden index, the Clinical diagnostic threshold of STAT3 protein for NSCLC was $76.38 \rho \mathrm{g} / 1$; under the cut-off point, the sensitivity $(S e)$ was $77.50 \%$, and the specificity $(S p)$ was $72.50 \%$.

\subsection{The Diagnostic Value of the Four Joint Detections (STAT3 + CEA + CA125 + CYFRA21-1)}

In parallel way, by drawing the ROC curve with statistical software SPSS17.0 according to the result of the expression of STAT3, CEA, CA125andCYFRA21-1 in serum, we got the $\mathrm{Az}$ of four joint detection was 0.930, and the Az of three joint detection of CEA, CA125 and CYFRA21-1 was 0.841, comparison between the two Az, $\mathrm{Z}=1.912, P=0.028$, the difference were statistically significant $(P<0.05)$, accuracy of the four Joint detection was significantly higher than the three Joint detection and it achieved a high diagnostic value.

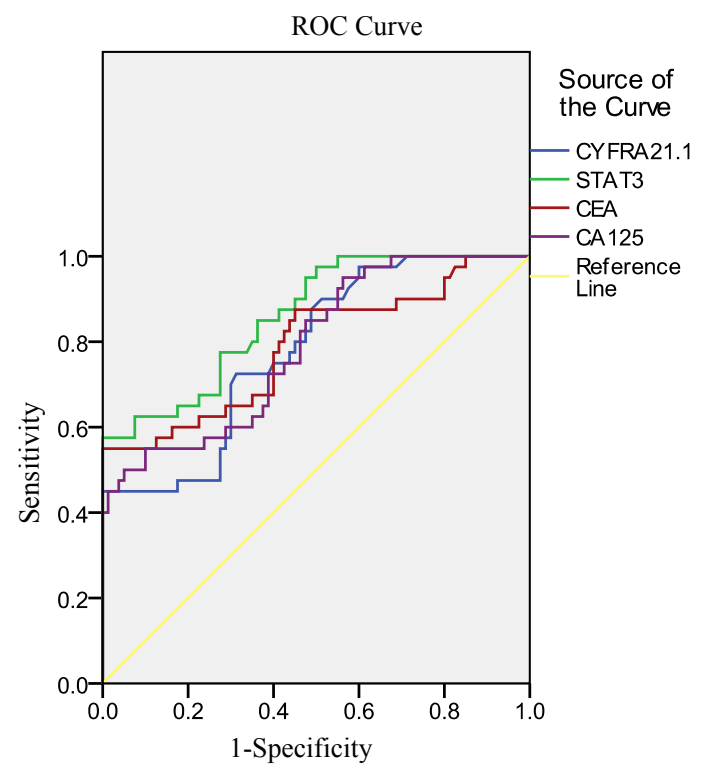

Figure 3. Az of STAT3 and CEA, CA125, CYFRA21-1.

Table 7. Comparison of the area under the ROC curve of STAT3, CEA, CA125, and CYFRA21-1.

\begin{tabular}{cccc}
\hline Index & $\mathrm{Az}$ & $Z$ value & $P$ value \\
\hline STAT3 & $0.860^{\mathrm{a}}$ & & \\
CEA & $0.789^{\mathrm{b}}$ & 1.203 & 0.115 \\
CA125 & $0.788^{\mathrm{c}}$ & 1.286 & 0.10 \\
CYFRA21-1 & $0.780^{\mathrm{d}}$ & 1.311 & 0.095 \\
\hline
\end{tabular}

Note: a compared with $\mathrm{b}, \mathrm{c}$ and d respectively, the difference was not statistically significant, $P>0.05$.

\section{DISCUSSION}

As the lack of specific clinical manifestations, the early diagnosis of lung cancer depends on the additional tests. At present, tumor markers have become an important part of laboratory examinations in early diagnosis of lung cancer. From the clinical point of view, tumor markers are the biochemical substances that can be measured, mainly distributed in the organization, plasma or other body fluids, and can prompt a kind of tumor when their presence upon to a certain level. Such as lung cancer is related to Carcinoembryonic antigen (CEA), carbohydrate antigen 125(CA125), Human cytokeratin fragment antigen 21-1(CYFRA21-1), neuron-specific enolase (NSE) and other markers, and the first three of them closer relationship with NSCLC, their joint has been widely used in clinical [1-4]. STAT3 is an important member of the STATs family, are defined as oncogenes [5]. Study found that STAT3's activationin can be observed in the development and progression of NSCLC, Its way through involved in the JAK/STAT3 signaling pathway mediated lung epithelial cell's proliferation and malignant transformation, preventing apoptosis, resulting in the occurrence of lung cancer [6,7]. Our study shown that STAT3's expression abnormally high in lung cancer cells and the blood of lung cancer patients either in genes or protein levels, which is consistent with the expression of the results reported, it suggesting that STAT3 is expected to become a new lung cancer markers for the characteristics that its content in tumor tissue is much higher than normal levels tissue or benign tumors, could be detected in the cells and the blood stability, and its concentration could reflect the degree of malignancy.

ROC curve is a very comprehensive, accurate and effective way for evaluating and testing target tumor markers in clinical application. According to Swets criterion, $\mathrm{Az}<0.5$, there is no diagnostic test value; $0.5<\mathrm{Az} \leq 0.7$, Less accurate; $0.7<\mathrm{Az} \leq 0.9$, have a certain accuracy and medium diagnostic value, can be recommended in clinical diagnosis; Az $>0.9$, higher accuracy and highervalue, can be used as an important diagnosis in clinical diagnosis. The results shown that, both the Az of STAT3 mRNA and STAT3 protein were between 0.7 and 0.9 , they had the same value for NSCLC diagnostic, both of which were moderate, and had no significant difference in accuracy, so both of them could be recommended for clinical. Compared to Real time PCR, ELISA is more stable to detect the level of STAT3 protein in serum, on the basis of ensuring the effectiveness, it's more simple, less expensive, more in line with the Practical principles of diagnostic tests, easier to apply in clinical, and we compared the Az of STAT3 protein with CEA, CA125, CYFRA21-1, respectively, the results shown that STAT3 could achieve the equal diagnosis value of each others, 
and no significant difference in accuracy. When comparing Se and Sp under the best cut-off point corresponding of four proteins, STAT3 is higher, $77.50 \%$ and $72.50 \%$, respectively. All above suggests that as a new marker associated with NSCLC, STAT3 has good prospects for clinical application.

The occurrence of Tumors is complex multi-stage pathological process, induced by multiple factors, a single tumor marker is difficult to objectively reflect this complex process, therefore, the combination of tumor markers has become a kind of method that commonly used in clinical, and have been a beneficial choice for diagnosis, treatment and prognosis prediction of lung cancer. The Az of the four joint detections (STAT3 protein, CEA, CA125 and CYFRA21-1) is 0.930, compared with the Az of the three joint (CEA, CA125 and CYFRA21-1), 0.841, the difference was statistically significant, it indicated that diagnostic accuracy of the new Joint Inspection method, composition of STAT3 and other three tumor markers, is improved significantly, Az $>0.9$, in addition, achieved a high diagnostic value, so it was expected to significantly improve the early diagnosis rate of NSCLC.

\section{OUTLOOK}

Early diagnosis of lung cancer is a problem long plagued in clinical, and finding a non-invasive safe, effective, practical and economical method of diagnosis is the goal pursued by many scholars over the years. This study is expected to provide a new cancer detection target and joint inspection methods to increase the current rate of early diagnosis of lung cancer. Meanwhile, the experiment opened up a new way of thinking for the research of STAT3 in the field of lung cancer.

\section{ACKNOWLEDGEMENTS}

This project was funded by the Sichuan Province Key Foundation of Science and Technology Agency (2006j13-192).

\section{REFERENCES}

[1] Stieber, P., Hasholzner, U., BodenmuJller, H., et al. (1993) CYFRA21-1. A newmarker in lung cancer. Cancer, 72, 707-713.

doi:10.1002/1097-0142(19930801)72:3<707::AID-CNC R2820720313>3.0.CO;2-X

[2] Henderson, R.A. and Finn, O.J. (1996) Human tumor antigens are ready to fly. Advances in Immunology, 62, 217-256. doi:10.1016/S0065-2776(08)60431-9

[3] Zhao, X., Wu, X.D., Xu, W.J., et al. (2011) Diagnostic value of combined detection of NSE, CYFRA2I-1, CEA and CA125 in lung carcinoma. Journal of Radioimmunology, 24, 696-697

[4] Niho, S. and Shinkai, T. (2001) Tumor markers in lung cancer. Japanese Journal of Cancer and Chemotherapy, 28, 2089-2093.

[5] Campbell, C.L., Jiang, Z., Savarese, D.M., et al. (2001) Increased expression of the interleukin-11 receptor and evidence of STAT3 activation in prostate carcinoma. American Journal of Pathology, 158, 25-32. doi:10.1016/S0002-9440(10)63940-5

[6] Liu, J. and Kern, J.A. (2002) Neuregulain-1 activates the JAK-STAT pathway and regulates lung epithelial cell proliferation. American Journal of Respiratory Cell and Molecular Biology, 27, 306-313. doi:10.1165/rcmb.4850

[7] Wang, F.-Y., Zhang, Y.-Q., Luo, M., et al. (2011) The expressions of p-Stat3, VEGF-C and MMP-2 in nonsmall cell lung cancer and their relationship with lymph node metastasis. Carcinogenesis, Teratogenesis \& Mutagenesis, 23, 107-122. 